\title{
Spin-Torque Oscillator using a Perpendicular Polarizer with Double Free Layers
}

\author{
Soo-Man Seo and Kyung-Jin Lee* \\ Department of Materials Science and Engineering, Korea University, Seoul 136-713, Korea
}

(Received 26 November 2008, Received in final form 18 December 2008, Accepted 18 December 2008)

\begin{abstract}
We conducted a micromagnetic modeling study to investigate the spin torque oscillator (STO) using a perpendicular polarizer. We used an additional layer of negative anisotropy constant materials (NAM) on a conventional STO. For the NAM layer, the magnetic easy plane is parallel to the in-plane easy axis of the free layer, and inhibits the development of the out-of-plane component of the magnetization in the free layer. As a result, this new type of STO provides a high frequency limit up to $50 \mathrm{GHz}$.
\end{abstract}

Keywords : spin transfer torque, spin torque oscillator, anisotropy energy, negative anisotropy

\section{Introduction}

The spin transfer torque (STT) [1] caused by the transfer of the spin angular momentum from the spinpolarized conduction electron to the local magnetization has attracted much attention in terms of its application and fundamental physics $[2,3]$. In the magnetic multilayer system, the STT acts similarly to an anti-damping torque on the magnetization and excites spin waves [4], which leads to current-induced magnetic switching [5].

When the intrinsic damping torque is compensated by STT, the local magnetization can sustain its precession motion. This steady motion of the magnetization provides a new method for applications of spintronic devices. One possible application is the wide-band tunable radio frequency (RF) oscillator for the application of telecommunication or logic devices, i.e. so-called spin torque oscillators (STO). From theory [6] and experiments [7], the current-induced precession can be achieved even without an external field by using the perpendicular polarizer as a pinned layer. Another example is the microwave assisted magnetic recording [8]. This concept requires several tens of $\mathrm{GHz}$ to gain the saturation digital recording at fields significantly below the medium coercivity. This is because the frequency of the ac field generated by the STO should be about the Larmor frequency, which is proportional to the coercivity of the medium. For instance, for a medium with a coercivity (or

*Corresponding author: Tel: +82-2-3290-3289

Fax: +82-2-928-3584, e-mail: kj_lee@korea.ac.kr the anisotropy field) of about $2 \mathrm{~T}$, the needed frequency is $50 \mathrm{GHz}$

According to the theoretical work [6], the angle between the precessing magnetization and the precession axis becomes smaller as the current (i.e. STT from the perpendicular pinned layer) increases. Therefore, the highest frequency obtained in this structure is determined by the current which provides the out-of-plane saturation of the magnetization in the free layer (i.e. the tiling angle with respect to the effective field $=0$ ). Since a higher frequency is better for the microwave assisted recording, this fundamental limitation should be resolved. In this work, we introduce negative anisotropy materials (NAM) for the high frequency excitation. The magnetic anisotropy energy is defined as follows,

$$
E_{K}=K \sin ^{2} \theta
$$

where $\theta$ is the angle between the local magnetization and the magnetic easy axis. For the conventional design of STO, the easy magnetization axes of the free layer lie on the film plane ( $x y$-plane of Fig. 1). From the definition of the anisotropy energy (Eq. (1)), for a negative $K$ value, the anisotropy energy $\left(E_{K}^{N A M}\right)$ of the NAM layer should be minimized (maximized) for $\theta=0^{\circ}\left(90^{\circ}\right)$. Thus, the magnetization $\left(\vec{M}^{N A M}\right)$ of the NAM layer energetically prefers to sustain its direction within the $x y$-plane. Also, it can be expected that $\vec{M}^{\text {NAM }}$ oscillates along the z-axis, since $E_{K}^{N A M}$ is independent of the in-plane angle. Several NAMs have previously been experimentally reported, e.g. $\mathrm{CoFe}, \alpha$-FeC, NiAs-typed MnSb, and hep CoIr [9-12]. To explore the STO with NAM layer, we performed a micro- 
(a)

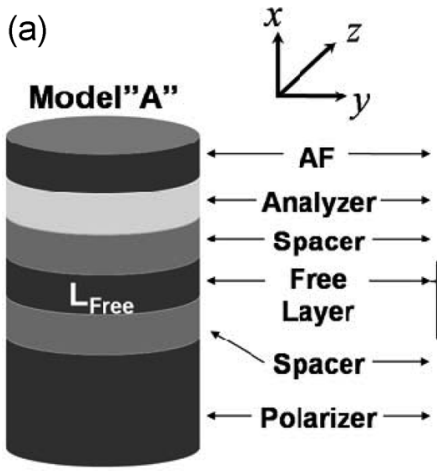

(c) $E_{K}^{\max }\left(\theta=90^{\circ}\right)$

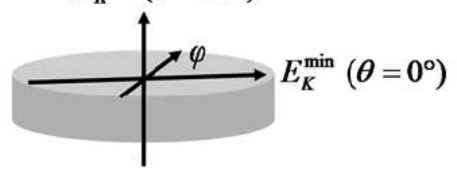

Fig. 1. Schematic diagram of modeling system. (a) Conventional STO, (b) STO with negative anisotropy materials (NAM) layer. (c) Angle dependence of anisotropy energy of magnetization with negative anisotropy constant. Here, $\theta$ and $\varphi$ correspond to the out-of-plane and in-plane angle, respectively.

magnetic simulation. In a comparison of the excited frequency of STO with and without the NAM layer, the current-driven precession frequency is obtained up to 50 $\mathrm{GHz}$.

\section{Micromagnetic Modeling}

To describe the effect of STT, the modified LandauLifshitz-Gilbert equation including additional torque terms is used,

$$
\begin{aligned}
\frac{\partial \mathbf{M}}{\partial t}= & -\gamma \mathbf{M} \times \mathbf{H}_{e f f}+\frac{\alpha}{M_{S}} \mathbf{M} \times \frac{\partial \mathbf{M}}{\partial t}-\frac{\gamma a_{J}}{M_{S}} \mathbf{M} \times(\mathbf{M} \times \hat{p}) \\
& -\frac{b_{J}}{M_{S}^{2}} \mathbf{M} \times\left(\mathbf{M} \times \frac{\partial \mathbf{M}}{\partial x}\right)
\end{aligned}
$$

where $\mathbf{M}$ is the local magnetization vector, $\gamma$ is the gyromagnetic ratio $\left(=1.76 \times 10^{7} \mathrm{sec}^{-1} \mathrm{Oe}^{-1}\right), \mathbf{H}_{\text {eff }}$ is the effective field consisting of the anisotropy, exchange, magnetostatic, and external field, $M_{S}$ is the saturation magnetization and $\alpha$ is the Gilbert damping constant. The coefficients $a_{J}$ and $b_{J}$ are given by $\hbar \eta_{j} / 2 e M_{S} d$ and $P j_{e} \mu_{B} /$ $e M_{S}$, respectively, where $\eta$ is the spin polarization coefficient, $j_{e}$ is the electrical current density, $e$ is the electron's electric charge, $P$ is the spin polarization, $d$ is the thickness of the free layer, and $\hat{p}$ is a unit vector along the direction of the magnetization of the pinned layer. Here, $a_{J}$ and $b_{J}$ represent the magnitude of the in-plane torque and the adiabatic spin torque (not the perpendicular torque or field like torque), respectively. The temperature is assumed to be zero. Fig. 1 shows a schematic diagram of the STO with the single free layer $\left(L_{\text {Free }}\right)$ (Model "A") and double free layers consisting of a conventional softmagnetic free layer and the NAM layer (Model "B"). Since the effect of STT appears on $L_{\text {Free }}$ and $L_{N A M}$, we considered the magnetization dynamics in these two layers. The nanopillar has a circular cross-section with the lateral dimensions of $35 \times 35 \mathrm{~nm}^{2}$ and the thickness of each free layer $\left(L_{F r e e}\right.$ and $\left.L_{N A M}\right)$ is $10 \mathrm{~nm}$. The unit cell of $35 \times 35 \times 1 \mathrm{~nm}^{3}$ is considered (i.e., the free layer consists of 10 cells along the thickness direction). Note that Slonczweiski's ST ( $a_{J}$ torque) is typically subjected on the bottom magnetization of the free layer. Since the $a_{J}$ torque is only applied on the bottom cell attached to the metallic spacer, there is a distribution of magnetization along the thickness direction in the free layer. Thus, it is reasonable to consider the $b_{J}$ torque induced by a gradient magnetization $(\nabla \mathbf{M})$, with the exception of the bottom cell of the free layer. We considered the typical soft ferromagnet for the conventional free layer. $\mathrm{Co}_{1-x} \mathrm{Ir}_{x}(x=0.2 \sim$ $0.3)$ is selected for the negative anisotropy materials [11]. The following materials parameters of $L_{\text {Free }}\left(L_{N A M}\right)$ are used: $1000(1600) \mathrm{emu} / \mathrm{cm}^{3}$ for $M_{S} ; 0\left(-1 \times 10^{7}\right)$ for $K$; $1.3 \times 10^{6}\left(1.3 \times 10^{6}\right) \mathrm{erg} / \mathrm{cm}$ for $A_{e x} ; 0.01(0.05)$ for $\alpha ; 0.7$ (0.2) for $P$; and $0.7(0)$ for $\eta$.

\section{Results and Discussion}

The excited frequency $(f)$ and the normalized magnetization $\left(M_{N}\right)$ as a function of current density $\left(j_{e}\right)$ are shown in Fig. 2(a) and Fig. 2(b), respectively. The solid
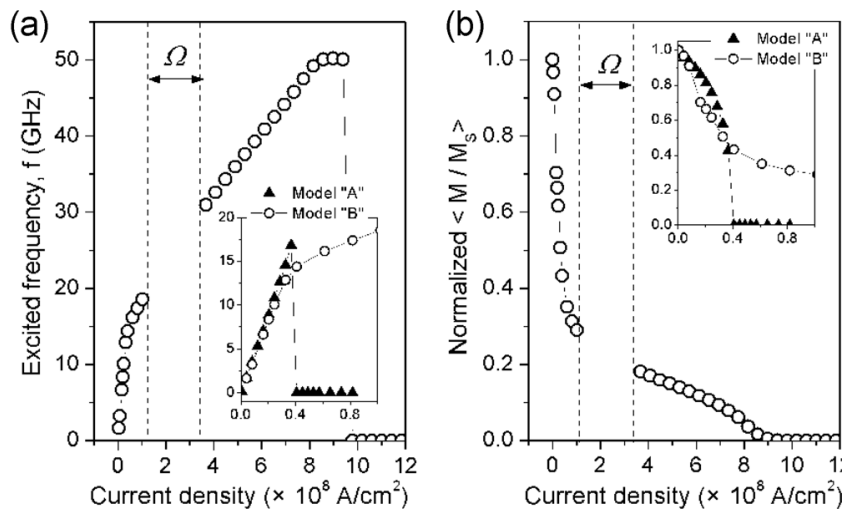

Fig. 2. (a) Excited frequency and (b) normalized magnetization as a function of current density. The solid triangle and open circle correspond to Model "A" and "B", respectively. For Model "B", $\Omega$ indicates the region where the eigen frequency does not appear. 

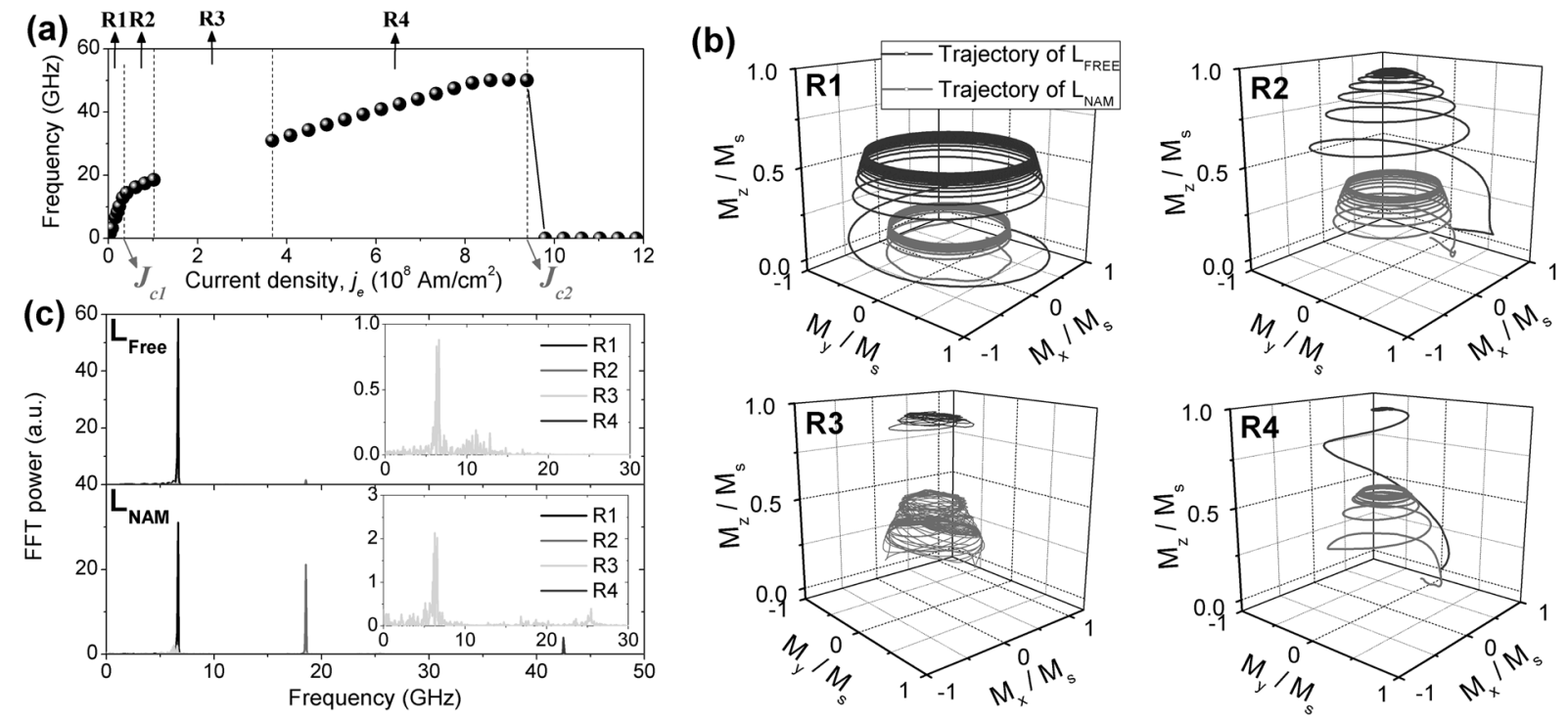

Fig. 3. (a) Excited frequency as a function of current density for model "B". Here, $J_{c 1}$ and $J_{c 2}$ indicate the critical current density for the saturation of $L_{F r e e}$ and $L_{N A M}$, respectively. (b) The typical trajectory of precession motion for R1 R4 is denoted in Fig. 3(a). Blue and red lines indicate the trajectory of the magnetization of $L_{F r e e}$ and $L_{N A M}$, respectively. (c) Fast Fourier transform (FFT) spectra of R1 R4. Especially, insets correspond to the case of R2.

triangle and open circle correspond to Model " $\mathrm{A}$ " and "B", respectively. As was theoretically predicted [4], the linear relationship between $f$ and $j_{e}$ is shown for model "A". For $j_{e}=4 \times 10^{7} \mathrm{~A} / \mathrm{cm}^{2}\left(=J_{c l}\right)$, a maximum $f\left(f^{\max }\right)$ of $16.8 \mathrm{GHz}$ is obtained and $M_{N}=0$ (Inset of Fig. 2(b)). Above this critical $j_{e}\left(j_{c}\right)$, the magnetization of the free layer is saturated at the $z$-axis and therefore no more precession is achieved at above the $f^{\max }$.

For model "B", below $J_{c 1}$, the relationship between $f$ and $j_{e}$ is similar to that of model "A" (Inset of Fig. 2(b)). We can then see a reduced slope from $J_{c 1}$ and $J_{c 2}(9.38 \times$ $10^{8} \mathrm{~A} / \mathrm{cm}^{2}$ ). At $J_{c 2}, f^{\max }$ is $50.3 \mathrm{GHz}$, which is three times larger than $f^{\max }$ model " $\mathrm{A}$ ". In particular, in the range of $j_{e}$ $=1 \sim 3.6 \times 10^{8} \mathrm{~A} / \mathrm{cm}^{2}$ (denoted as $\Omega$ ), there is no eigen frequency for the precession motion.

In order to gain a further understanding of the various excited modes of model "B", we investigated the trajectory of the precession motion. Fig. 3(a) shows the excited frequency as a function of current density for model "B". $J_{c 1}$ and $J_{c 2}$ indicate the critical current density for the saturation of $L_{\text {Free }}$ and $L_{N A M}$, respectively. According to the dependence between $f$ and $j_{e}$, the typical trajectory of the precession motion is observed (Fig. 3(b)). In Fig. 3(b), the trajectories of $L_{\text {Free }}$ and $L_{N A M}$ are shown. First, in the range of $0 \sim J_{c 1}$ ("R1" in Fig. 3(b)), it is observed that $L_{F r e e}$ and $L_{N A M}$ coherently precess. From the fast Fourier transform (FFT) spectra of the magnetizations in this range of the current, we can see the single peak (black line peak in Fig. 3(c)). The normalized $z$-compo- nent $\left(M_{Z} / M_{S}\right)$ of $L_{\text {Free }}$ is larger than $L_{N A M}$, since the $a_{J}$ torque only exerts on the bottom layer of $L_{\text {Free }}$. Second, in the range of $J_{c 1} \sim 1 \times 10^{8} \mathrm{~A} / \mathrm{cm}^{2}$, the slopes of $f$ and $j_{e}$ are reduced. For this range of the current density, we can see that $L_{\text {Free }}$ is entirely saturated ("R2" in Fig. 3(b)). From the FFT spectra (red line in Fig. 3(c)), the peak of $L_{\text {Free }}$ is close to zero, but the peak of $L_{N A M}$ shows a nonzero value. For the range of $1 \sim 3.6 \times 10^{8} \mathrm{~A} / \mathrm{cm}^{2}$ ("R3" in Fig. $3(\mathrm{~b})$ ), the two layers incoherently rotate. Widely distributed FFT spectra were shown (Insets of Fig. 3(c)). For the range of $3.6 \times 10^{8} \sim J_{c 2}$ ("R4" in Fig. 3), the behavior of the two layers is similar to the case of R2. In both cases of R2 and R4, the excited frequency is entirely a result of the precession of the magnetization of $L_{N A M}$, since the magnetization of $L_{\text {Free }}$ is almost saturated towards the direction of the effective field (z-axis). However, for the case of "R4", the magnetization of $L_{\text {Free }}$ is more rapidly saturated in the z-axis, and the tilting angle of $L_{N A M}$ is larger than the case of "R2". Above $J_{c 2}$, the magnetization of the two layers is entirely saturated, and there is therefore no precessional motion of the magnetization.

\section{Conclusion}

In conclusion, we have investigated the STO using a perpendicular polarizer. We showed the enhanced frequency for the STO with NAM layers. Since the magnetization of the NAM layer prefers an in-plane direction due to the negative anisotropy constant, the NAM layer acts 
similarly to an energy barrier for the out-of-plane saturation of $L_{\text {Free }}$. As a result, by inserting a NAM free layer, the critical current density for the saturation of $L_{\text {Free }}$ and the oscillation frequency are enhanced. Our results indicate that it is possible to increase the precession frequency by introducing the NAM since it inhibits the outof-plane saturation of the magnetization in the free layer. This high precession frequency can be used for the STO in the microwave assisted magnetic recording even without heating the medium.

\section{Acknowledgements}

This research was supported by a grant from the Fundamental R\&D Program for Core Technology of Materials funded by the Ministry of Commerce, Industry and Energy, Republic of Korea.

\section{References}

[1] J. C. Slonczewski, J. Magn. Magn. Mater. 159, L1 (1996); L. Berger, Phys. Rev. B 54, 9353 (1996).

[2] S. I. Kiselev et al., Nature 425, 380 (2003).

[3] S.-W. Jung, and H.-W. Lee, J. Magnetics 12(2), 1 (2007).

[4] Joo-Von Kim et al., J. Magnetics 12(2), 53 (2007).

[5] E. B. Myers et al., Science 285, 867 (1999).

[6] K.-J. Lee, O. Redon, and B. Dieny, Appl. Phys. Lett. 86, 022505 (2005).

[7] D. Houssameddine et al., Nat. Mater. 6, 447 (2007).

[8] J.-G. Zhu, X. Zhu, and Y. Tang, IEEE Trans. Magn. 44, 125 (2008).

[9] M. Takahashi and S. Kadowaki, J. Phys. Soc. Jpn. 48, 1391 (1980).

[10] M. Takahashi, Y. Takahashi, and H. Shoji, IEEE Trans. Magn. 37, 2179 (2001).

[11] N. Kikuchi et al., J. Phys. Condens. Matter 11, L485 (1999).

[12] T. Oikawa et al., IEEE Trans. Magn. 38, 1976 (2002). 